\title{
Two New Species of Geranium in Japan.
}

\author{
by
}

\section{J. Matsumura.}

Geranium shikokianum, Matsum. sp. nov.

Planta usque $64 \mathrm{~cm}$. alta. Radix...Multicaulis, suberectus; caulis ad nodos subincrassatus, sursum pilis patentibus v. subreflexis vestitus, glandulis nullis, deorsum glabrescens lucidus. Folia radicalia........, caulina longe petiolata, petiolis usque $18 \mathrm{~cm}$. longis sursum hirsutis, stipulis ampliis connatis membranaceis, badiis late ovatis v. ellipticis, obtusis v. cuspidatis saepe sublobatis, glabris maryine saepe ciliolatis; lamina ambitu reniformia, ultra medium palmato- 5-7- fida v. partita, segmentis cuneatis, acutis, 2-3incisis, supra adpresso-puberula, subtus ad venas parce hirsutula. Pedunculi folia mutities superantes usque $10 \mathrm{~cm}$. longi, pilis patentibus v. subreflexis vestiti, pedicellis fructiferis declinatis, usque $2 \frac{1}{2} \mathrm{~cm}$. longis, bracteis membranaceis lineari-oblongis obtusis v. acutis glabris instructi. Flores magni; sepala oblonga, aristata, 5-nervia, extus parce puberula v. glabrescentia, intus glabra, $9 \mathrm{~mm}$. longa, $4 \mathrm{~mm}$. lata ; petala sepalis duplo longiora, obovata, roseo-purpurea, 7-nervia, integra v. cuspidata, $2 \mathrm{~cm}$. longa, prope basin ciliata v. plus minus barbata. Filamenta dilatata stylos subaequantia, partibus dilatatis ciliatis; antherae ellipticae glabrae. Ovarium hirsutum, stylis liberis, fere a basi ad apicem stigmatosis. Fructus usque $28 \mathrm{~mm}$. longus, valvis puberulis, apice basique longe ciliato-barbatis. Semina ellipsoidea, opaca, minutissime impressopunctata.

A. G. Sieboldi, Maxim. indumento, stipnlis ampliis connatis facile distinguenda.

Hab. in Japonia: insula Shikoku, montibus Ishizuchi et Tebako leg. S. Yano anno 1890. Fl. et fr. Augusto.

Geranium hakusanense, Matsum. sp. nov.

Planta $37 \mathrm{~cm}$. alta, Radix...... Caulis suberectus, angulatus sub lente pilis minutis adpresso-reflexis conspersus. Folia radicalia......, caulina modice, superiora brevissime petiolata, petiolis patentibus, subcanescentibus, stipulis 
ovatis acutis herbaceis, glabrescentibus integerrimis; lamina ambitu reniformia, fere ad basin palmato-5-partita, segmentis cuneatis acute angusteque 3-5-incisis, laciniis ultimis lancenlatis acutis, supra minutissime puberula, subtus pallidiora ad venas tantum adpresse hirsutula. Pedunculi folia multo superantes, pedicellis fructiferis cernuis, bracteis ovatis acuminatis instructi. Flores $3 \frac{1}{2} \mathrm{~cm}$. in diametro ; sepala oblonga v. ovato-elliptica, aristata, 6nervia, extus ad nervos adpresse puberula, intus glabra; petala purpurea, sepala multo superantia, obovata, integra, intus ad basin barbata, supra basin pilosa. Filamenta basi abrupte dilatata ciliata, stylos subaequantia ; antherae ellipticae. Ovarium villosum, stylis liberis tantum supra medium stigmatosis. Fructus $3 \frac{1}{2} \mathrm{~cm}$. longus, valvis puberulis, apice basique hirsutis. Semina ovalia, opaca, minutissime impressopunctata. G. pratense, Iinuma Soomoku-zusetsu XII, t. 45. (Hakusan-fūro). A. G. Sieboldi, Maxim. cui affinis, differt foliorum laciniis acutioribus, caulibus foliisque fere glabrescentilus, stipulis connatis majoribrs, petalis pilosis.

Hab. in japonia: prov. Shinano, monte Asama leg. C. Owatari anno 1894. Fl. et fr. Augusto.

Observations on the Flora of Japan.

(Continued fiom p. 118.)

By

T. Makino,

Assistant in the Botanical Institule, Science

Coliege, Imperial University of Tohyo.

Rumex (Lapathum) Daiwoo (Sieb.) Makino nom. nov.

Robust perennial, attaining about $1 \frac{2}{3} \mathrm{~m}$. in height. Roots thick, yellow. Stem stout, erect, simple, flexuous, striato-sulcate, fistulous, glabrous, but often pubescent under the nodes, often tinged with rose-purple colonr. Leaves alternate, ample, petioled, gradually diminishing in size and transferring into bracts, oblong-ovate, but ovate-lanceolate in the above ones, broadly ovate to ovato-oblong in the radical ones, obtuse or acutish, broadly 